Short communication

\title{
Optimal spherical spline filters for the analysis and comparison of regional-scale tomographic models
}

\author{
Andreas Fichtner ${ }^{\mathrm{a}, *}$, Stewart Fishwick ${ }^{\mathrm{b}}$, Kazunori Yoshizawa ${ }^{\mathrm{c}}$, Brian L.N. Kennett ${ }^{\mathrm{d}}$ \\ ${ }^{a}$ Department of Earth Sciences, Utrecht University, Utrecht, The Netherlands \\ ${ }^{\mathrm{b}}$ Department of Geology, University of Leicester, Leicester, United Kingdom \\ ${ }^{\mathrm{c}}$ Department of Natural History Sciences, Faculty of Science, Hokkaido University, Sapporo, Japan \\ ${ }^{\mathrm{d}}$ Research School of Earth Sciences, The Australian National University, Canberra, Australia
}

\section{A R T I C L E I N F O}

\section{Article history:}

Received 14 August 2011

Received in revised form 28 October 2011

Accepted 2 November 2011

Available online 10 November 201110

November 2011

Edited by George Helffrich

\section{Keywords:}

Seismic tomography

Spatial filtering

Spherical splines

Abel-Poisson kernels

Model comparison

\begin{abstract}
A B S T R A C T
Advances in seismic tomography lead to increasingly detailed models of the Earth that are often represented on irregular and resolution-adaptive grids. To take full advantage of such models, their assessment must progress beyond a purely visual analysis, and tools must become available for their quantitative comparison.

We present a method for the spectral analysis and comparison of multi-scale tomographic models. The method is applicable to irregular grids on the sphere, and is more efficient that filters based on sphericalharmonic expansions or convolution integrals. The combination of a spherical spline representation of tomographic information with Abel-Poisson scaling enables the construction of targetted spatial filters by solving a nonlinear inverse problem for appropriate weighting coefficients. This can be readily achieved with a simulated annealing approach for the limited number of weights. Once suitable filters have been generated they can be employed to address issues such as the patterns of small-scale heterogeneity, transitional structures and comparison of independent models from a region.

We illustrate our method in a series of applications where we use different bandpass filters to detect differences in the distribution of small-scale heterogeneity beneath central and eastern Europe, and to compare several recent tomographic models of the Australian region.
\end{abstract}

(C) 2011 Elsevier B.V. All rights reserved.

\section{Introduction}

Improvements in tomographic techniques and data coverage continue to increase the resolution of tomographic images, and the combination of teleseismic and regional data allows us to constrain heterogeneities with length scales that differ by several orders of magnitude. These advances have led to the widely recognised need to progress beyond a purely visual analysis in order to exploit the vast amount of information contained in highresolution multi-scale models. Particular challenges include: (1) the identification of the size and distribution of small-scale heterogeneities that are embedded within regions of consistently high or low velocities, where colour scales tend to saturate; (2) the quantification of the sharpness of slow-to-fast transitions; (3) the assessment of scale-dependent similarities of independently derived tomographic images, especially in the context of recent attempts to construct regional 3D reference models, and (4) the regional-scale spectral analysis on adaptive irregular grids that prevent the use of spherical harmonic expansions.

\footnotetext{
* Corresponding author.

E-mail address: fichtner@geo.uu.nl (A. Fichtner).
}

Previous analysis of some of these questions has involved a variety of techniques. Simple calculations of the horizontal gradient in shear velocity has been used to demarcate sharp transitions in structure, unbiased by choices in reference model, colour scale and saturation of the tomographic image itself (Fishwick, 2006). This approach has been used at both continental scale (e.g. Fishwick et al., 2008) and for more local-regional tomographic studies (e.g. Lei et al., 2009). Comparison of different tomographic models through gradient maps remains challenging due to the influence of the choice of any particular parameterisation and regularisation. Hernlund and Houser (2008) compare different tomographic models of both $\mathrm{P}$ and $\mathrm{S}$ velocity through an assessment of their statistical properties. Their study, focussed on the deep mantle, illustrates that the nature of the distributions with different reference velocities, peak amplitudes, and standard deviations makes it difficult to assess how, where and why the different models differ in velocity structure. The distributions do, however, suggest a strong decoupling between $\mathrm{P}$ and $\mathrm{S}$ velocity in the deep mantle; an observation recently tested with synthetic modelling (Della Mora et al., 2011). Becker and Boschi (2002) compared a variety of global tomographic models through an expansion into spherical harmonics. The spectral signal and radial correlation of 
the individual models were computed, and then spectral correlations were performed to compare the different models. A potential problem in assessing the shortest wavelength structure is that the expansion of block models into spherical harmonics can lead to spurious power, although Becker and Boschi (2002) conclude that this effect is small for degrees $\ell \leqslant 20$. Clearly, such an approach would be considerably less accurate for models based on adaptive irregular grids, and for regional models that are not defined on the whole sphere. Most recently, Simons et al. (2010) proposed spherical wavelet bases that can be used for both the analysis and the construction of sparse tomographic models with spatially variable resolution.

In this short note we present a novel approach for the construction of spatial bandpass filters with pre-defined properties that can be used to address the challenges in tomographic model analysis we have mentioned above. The method is well-suited for models defined on irregularly spaced grid points, and it does not require special computational resources. Our approach is more efficient than filtering based on spherical harmonic expansions that may require prohibitively large numbers of coefficients, e.g. when the grid spacing is locally refined to well below $1^{\circ}$. Following the description of the method itself (Section 2) we illustrate its application to the spectral-analysis and comparison of regional-scale tomographic models (Section 3).

\section{Bandpass filtering of tomographic images on sections of the sphere}

Our method proceeds in three steps: The representation of a tomographic model at one particular depth in terms of spherical splines, followed by the design and application of a bandpass filter that suits a specific purpose.

\subsection{Spherical spline approximation}

Following Freeden et al. (1998), we start our development with the representation of a scalar quantity $v$, such as a seismic velocity or density at a specific depth, in terms of spherical splines. Without loss of generality we assume $v$ to be given at $N$ grid points $\mathbf{x}_{1}, \ldots, \mathbf{x}_{N}$ on the unit sphere $\Omega$. The grid points need not be regularly spaced. From the discrete set of points we obtain a continuous representation by interpolation with spherical splines

$\bar{v}(\mathbf{x})=\sum_{i=1}^{N} v_{i} K_{h}\left(\mathbf{x}_{i}, \mathbf{x}\right), \quad \mathbf{x} \in \Omega$,

where the Abel-Poisson kernel

$K_{h}(\mathbf{x}, \mathbf{y})=\frac{1}{4 \pi} \frac{1-h^{2}}{\left(1+h^{2}-2 h \mathbf{x} \cdot \mathbf{y}\right)^{3 / 2}}, \quad \mathbf{x}, \mathbf{y} \in \Omega$

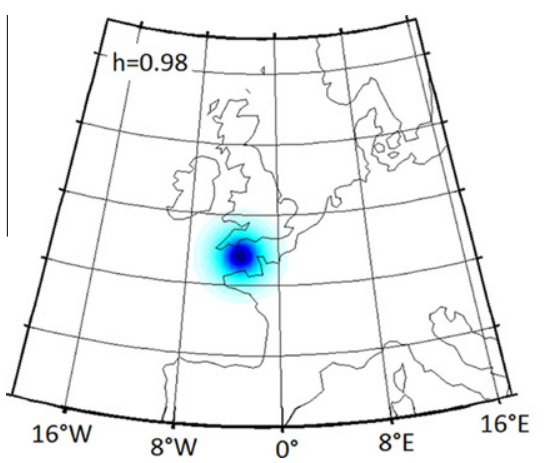

with the scaling parameter $h \in(0,1)$ is chosen as spline basis. AbelPoisson kernels for different values of $h$ are illustrated in Fig. 1. The $N$ interpolation conditions

$v\left(\mathbf{x}_{k}\right)=\bar{v}\left(\mathbf{x}_{k}\right)=\sum_{i=1}^{N} v_{i} K_{h}\left(\mathbf{x}_{i}, \mathbf{x}_{k}\right), \quad k=1, \ldots, N$,

yield a linear system of equations with a unique solution for the spline coefficients $v_{i}$.

\subsection{Lowpass filtering of spherical splines}

With the tomographic images expressed in terms of spherical splines, we proceed with the construction of a spatial lowpass filter. In general, the spatial filtering of the spline is achieved via a spherical convolution of $\bar{v}(\mathbf{x})$ with a scaling function $\varphi$ :

$(\varphi * \bar{v})(\mathbf{x})=\int_{\Omega} \varphi(\mathbf{x} \cdot \mathbf{y}) \bar{v}(\mathbf{y}) d^{3} \mathbf{y}$.

Since we work on the unit sphere, $\varphi$ is defined on the interval $[-1,1]$. We can characterise the action of the filter by expanding $\bar{v}(\mathbf{x})$ in spherical harmonics:

$$
\begin{aligned}
(\varphi * \bar{v})(\mathbf{x}) & =\sum_{n=0}^{\infty} \sum_{j=0}^{2 n+1} v_{n, j} \int_{\Omega} \varphi(\mathbf{x} \cdot \mathbf{y}) Y_{n, j}(\mathbf{y}) d^{3} \mathbf{y} \\
& =\sum_{n=0}^{\infty} \sum_{j=0}^{2 n+1} v_{n, j} \varphi_{n} Y_{n, j}(\mathbf{x}),
\end{aligned}
$$

where $\varphi_{n}$ are the Legendre coefficients of the scaling function $\varphi$. Thus, upon convolving $\bar{v}(\mathbf{x})$ with $\varphi$, the spherical harmonic coefficients of a specific degree $n$, i.e. $v_{n, j}$, are weighted by the Legendre coefficient $\varphi_{n}$. In anticipation of the following applications, we focus our attention on the Abel-Poisson scaling function

$\varphi(t)=\frac{1}{4 \pi} \frac{1-g^{2}}{\left(1+g^{2}-2 g t\right)^{3 / 2}}, \quad g \in(0,1)$,

the Legendre coefficients for which are given by $\varphi_{n}=g^{n}$. Since the filter parameter $g$ ranges between 0 and 1, the Abel-Poisson scaling function acts as a lowpass, meaning that large spherical harmonic degrees are down-weighted relative to smaller ones. The most advantageous property of the Abel-Poisson scaling function is, that its spherical convolution with a spherical spline can be computed without explicitly solving the integral in Eq. (4). Indeed, we have (see Appendix A):

$(\varphi * \bar{v})(\mathbf{x})=\sum_{i=1}^{N} v_{i} K_{g h}\left(\mathbf{x}_{i}, \mathbf{x}\right)$

Eq. (7) means that the lowpass filtering can be achieved simply by changing the scaling parameter of the Abel-Poisson kernel from $h$ to the smaller value $g h$.
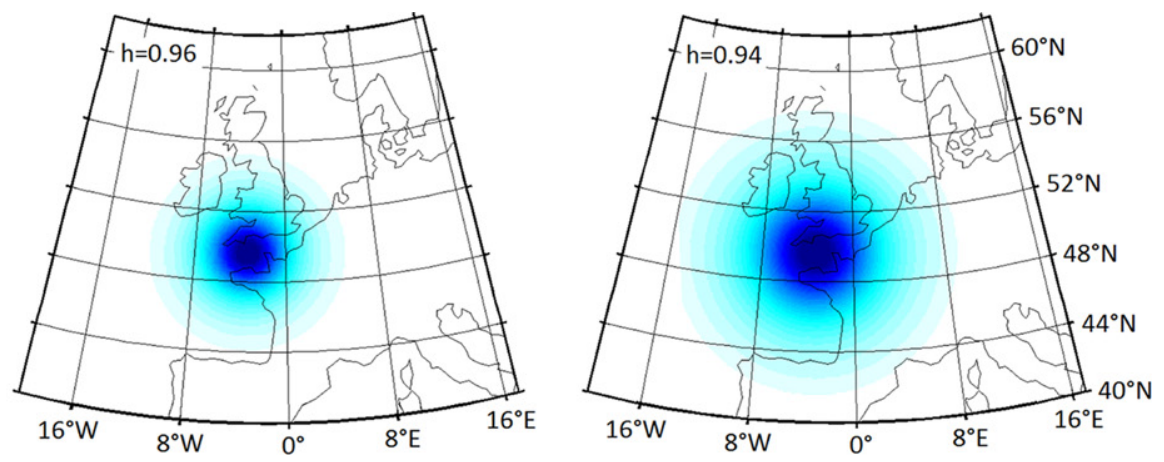

Fig. 1. Abel-Poisson kernels for different scaling parameters $h$ 


\subsection{Construction of optimal bandpass filters}

We can now construct computationally efficient bandpass filters by forming linear combinations of few Abel-Poisson scaling functions with different parameters $g$ :

$\varphi(t)=\sum_{k=1}^{M} a^{(k)} \varphi^{(k)}(t)$

Since the spherical convolution is linear in $\varphi$, the bandpass filter can effectively be applied by just adding weighted lowpass filtered versions of the spherical spline:

$(\varphi * \bar{v})(\mathbf{x})=\sum_{k=1}^{M} a^{(k)}\left(\varphi^{(k)} * \bar{v}\right)(\mathbf{x})$.

One lowpass filter $\varphi^{(k)}$ modifies the spherical harmonic coefficients of the spline $\bar{v}$ from $v_{n, j}$ to $a^{(k)}\left(g^{(k)}\right)^{n} v_{n, j}$, and the total effect of the linearly combined lowpass filters is then

$v_{n, j} \rightarrow f_{n} v_{n, j}, \quad f_{n}=\left[\sum_{k=1}^{M} a^{(k)}\left(g^{(k)}\right)^{n}\right]$.

The $2 M$ parameters $a^{(k)}$ and $g^{(k)}$ with $k=1, \ldots, M$, can now be chosen such that the filter coefficients $f_{n}$ approximate a pre-defined sequence $f_{n}^{(0)}$ of target-coefficients: $f_{n}^{(0)} \approx f_{n}$. An optimal set of parameters in the $L_{2}$ norm sense can be found by minimising the objective functional

$\chi=\sum_{n=0}^{n_{\max }}\left[f_{n}^{(0)}-\sum_{k=1}^{M} a^{(k)}\left(g^{(k)}\right)^{n}\right]^{2}$,

where $n_{\max }$ is the maximum spherical harmonic degree involved in the optimisation. Typically $n_{\max }$ is significantly larger than the maximum degree contained in the tomographic images. Since $\chi$ is highly non-linear with respect to $a^{(k)}$ and $g^{(k)}$, the optimisation problem is solved most efficiently using global methods of the Simulated Annealing type (Kirkpatrick et al., 1983). An example of a 20parameter $(M=10)$ optimal filter with a pass band between $2^{\circ}$ and $5^{\circ}$ is shown in Fig. 2.

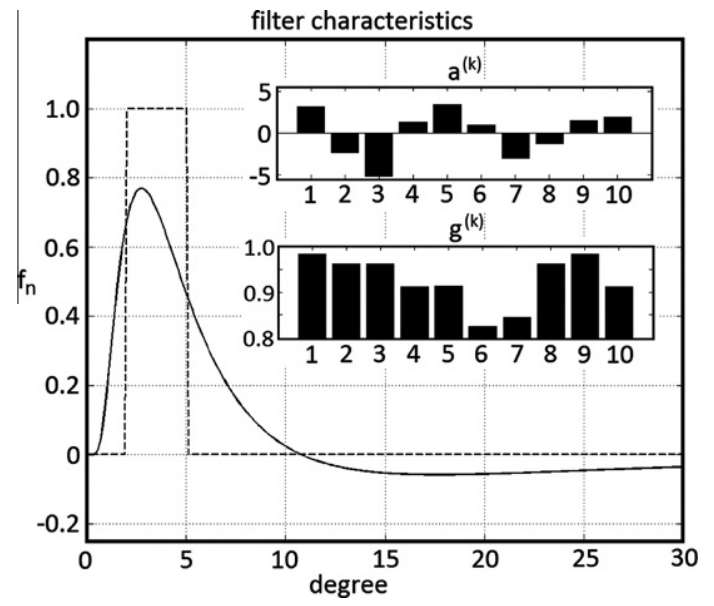

Fig. 2. Coefficients of a bandpass filter with 20 parameters $(M=10)$ plotted with respect to the spatial wavelength of spherical harmonics. The target coefficients, plotted as dashed curve, are equal to 1 between $2^{\circ}$ and $5^{\circ}$, and zero elsewhere. The optimal coefficients, obtained by minimising the $L_{2}$ misfit $\chi$ from Eq. (11) are shown as solid curve. The optimal parameters $a^{(k)}$ and $g^{(k)}$ are illustrated by the bar diagrams.

\section{Illustrations}

This approach to spatial bandpass filtering is useful in a variety of applications. These include the identification of small-scale heterogeneities that are difficult to distinguish visually, the quantification of the sharpness of apparently rapid transitions, the spectral analysis of tomographic models, and the comparison of tomographic models that aims at the identification of robust structural features. To illustrate the application of the optimal bandpass filtering, we consider the spectral analysis of a European uppermantle model (Section 3.1) and the comparison of three independently derived mantle models of Australia (Section 3.2).

\subsection{Spectral analysis of tomographic models with resolution-adaptive parametrisation}

We start with the spectral analysis of a European mantle model, shown in Fig. 3a, and previously discussed by Fichtner and Trampert (2011). The model was derived using a full waveform tomography approach as described in Fichtner et al. (2009). To prevent the occurence of artefacts, the parametrisation is adapted to a priori estimates of resolution. Beneath the densely covered Mediterranean, for instance, the horizontal grid spacing is $0.5^{\circ}$, whereas a $1.0^{\circ}$ spacing is chosen beneath northern Africa where less data are available. Each of the irregularly spaced model grid points serves as collocation point in the spherical spline interpolation of Eq. (1). The Hellenic slab, the Iceland plume and the Tornquist-Teisseyre Zone (TTZ) appear among the most prominent features at $100 \mathrm{~km}$ depth.

To identify the distribution and strength of small-scale heterogeneities, as well as the sharpness of the visually prominent TTZ, we apply various bandpass filters, the characteristics of which are shown in Fig. 3b. The bandpass filters were designed to be as close to 1 within and as close to 0 outside the intervals $\left[2^{\circ}, 5^{\circ}\right]$, $\left[5^{\circ}, 10^{\circ}\right]$ and $\left[10^{\circ}, 50^{\circ}\right]$, respectively. The filtered images are shown in Fig. 3c.

Contrary to the visual impression from Fig. 3a, the TTZ does not appear as a dominant transition at short wavelengths between $2^{\circ}$ and $5^{\circ}$. Instead, the Hellenic slab is the dominant small-scale feature. The amplitude of the short wavelength anomaly that can be observed beneath the TTZ is similar in magnitude to features in western Europe and suggests that the TTZ is not unique in terms of the velocity contrast at this depth. In contrast, Precambrian Europe east of the TTZ is nearly homogeneous at wavelengths below $5^{\circ}$. One plausible explanation is that within this region, the lithosphere is consistently thicker than $100 \mathrm{~km}$ depth, and therefore the contrasting velocity associated with adjacent lithosphere and asthenosphere is not observed. Additionally, the lack of short wavelength anomalies suggests that contrasts in temperature and composition within the Precambrian lithosphere do not produce sufficiently large, and coherent, velocity perturbations to be observed as variations on length scales of $2^{\circ}$ to $5^{\circ}$. The same is true for the Iceland plume, although in this region the lack of velocity anomalies at wavelengths shorter than $5^{\circ}$ may be due to the spreading of the plume head as it approaches the surface.

\subsection{Comparison of tomographic models}

Over the past decades, seismic tomography advanced substantially thanks to improvements in both data and methods, often leading to a diversity of tomographic models for the same geographic region. While the long-wavelength features in these models often appear similar, their resemblance on shorter wavelengths can be hard to assess visually. This difficulty is most pronounced when small-scale heterogeneities are embedded 
original model

a

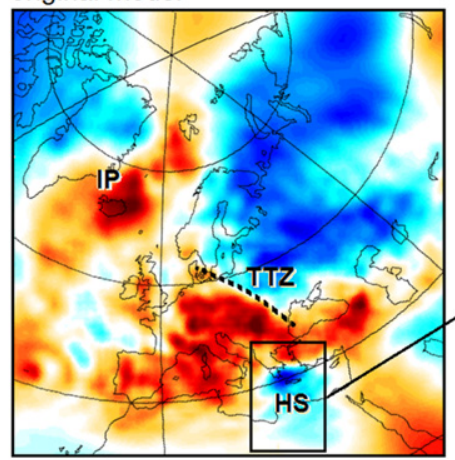

b

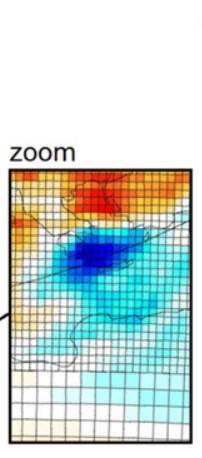

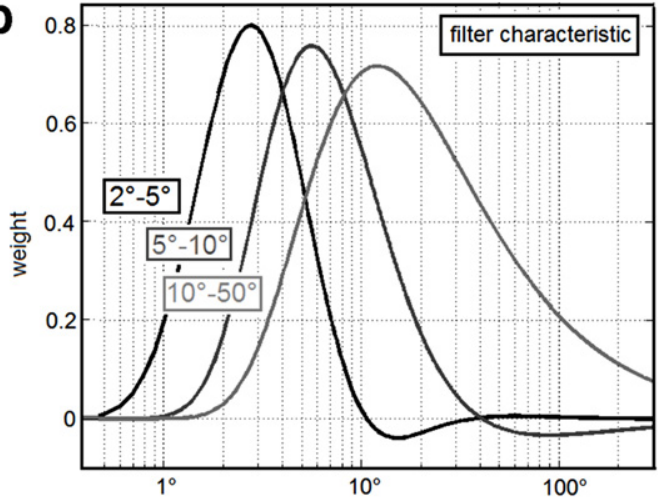

C

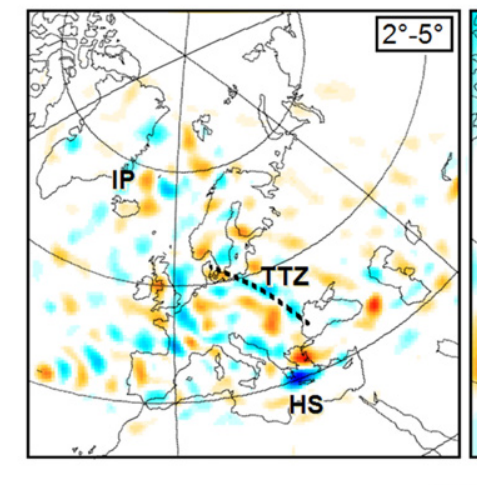

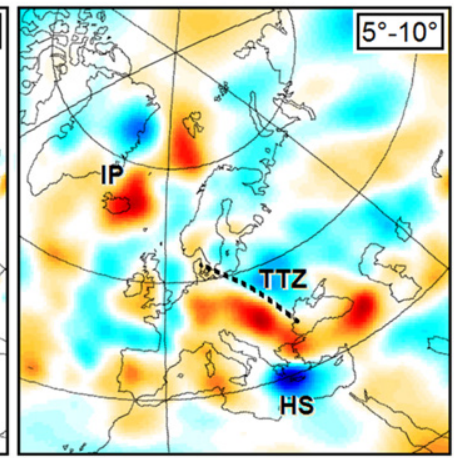

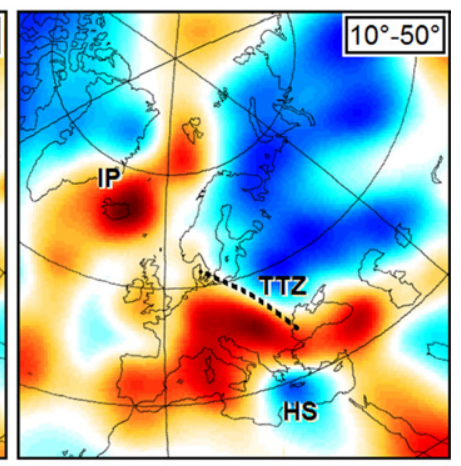

$+0.35$

$\Delta \mathrm{v}_{\mathrm{e}}\lceil\mathrm{km} / \mathrm{s}\rceil$

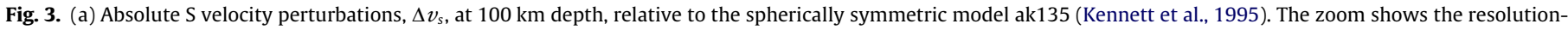

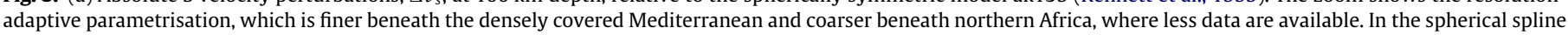

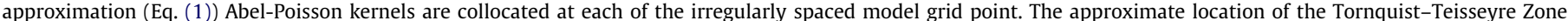

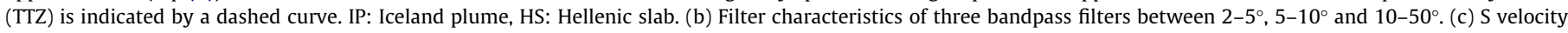
distributions filtered with the bandpasses shown in (b).

within large-scale regions of exceptionally low or high velocities where colour scales tend to saturate. The extent to which models differ as a function of scale, provides insight into the influence of various factors such as data selection and quality, errors in source estimates, regularisation and methodology. Recently, the need to identify the robust features of independent tomographic models on all scales has become increasingly pressing with attempts to construct regional 3D reference Earth models (e.g. Schivardi and Morelli, 2011; Molinari and Morelli, 2011; Kennett et al., 2011).

One of the most thoroughly investigated regions is the Australian continent where tomographic studies with a large variety of methods and data have led to an exceptional range of models, three of which are shown in the left column of Fig. 4 at the depth of $100 \mathrm{~km}$. The model of Fishwick et al. (2008) was constructed using the method of Cara and Lévèque (1987), starting with the construction of path-average $1 \mathrm{D}$ profiles that were subsequently mapped into a 3D tomographic image. Yoshizawa and Kennett (2004) recast the mapping procedure into a three-stage process working with intermediary phase speed maps, so that they can account for finite-frequency effects and departures from great-circle paths. Finally, the model by Fichtner et al. (2010) is based on a full waveform tomography approach using spectral-element simulations of seismic wave propagation combined with adjoint techniques. The iterative improvement of the model and the absence of an explicit regularisation are most likely responsible for the exceptional strength of the $\mathrm{S}$ velocity perturbations. Images of the different models bandpass filtered between $2-5^{\circ}, 5-10^{\circ}$ and $10-20^{\circ}$, respectively, are shown to the right of the original models in Fig. 4.
At the shortest wavelengths, from 2 to $5^{\circ}$, the models have few features in common. Even the location of low-velocity perturbations associated with the volcanic centres distributed along the Australian east coast, while apparently recognisable in the original models (left column of Fig. 4), is seen to be variable when filtered to the short wavelengths. All the models show short wavelength variability in velocity within the Precambrian regions of central and western Australia (in contrast to the observations in eastern Europe), however there is no consistent agreement on the exact location of these fast and slow anomalies.

At intermediate wavelengths, from 5 to $10^{\circ}$, the similarity between the different models is increased substantially. Structural features that are imaged consistently, independent of both method and data, include the sharp western edge of the Yilgarn and Pilbara cratons, the general zone of low velocities beneath NE Australia related to Cenozoic volcanism, and various low-velocity patches within Proterozoic central Australia that are imaged with different strengths.

As expected already from the visual inspection of the original images, the models are most similar at the longest wavelengths from 10 to $20^{\circ}$. In particular, the transitions from the low $S$ velocities of Phanerozoic eastern Australia to the high $S$ velocities of Precambrian Australia appear in nearly identical positions. It is at this level of filtering that the regional models approach the style of results from global inversions (e.g. Lebedev and van der Hilst, 2008; Ritsema et al., 2011).

The origins of the discrepancies between the tomographic models are difficult to quantify exactly. The appearance of elongated streaks within the models can be readily explained by the 

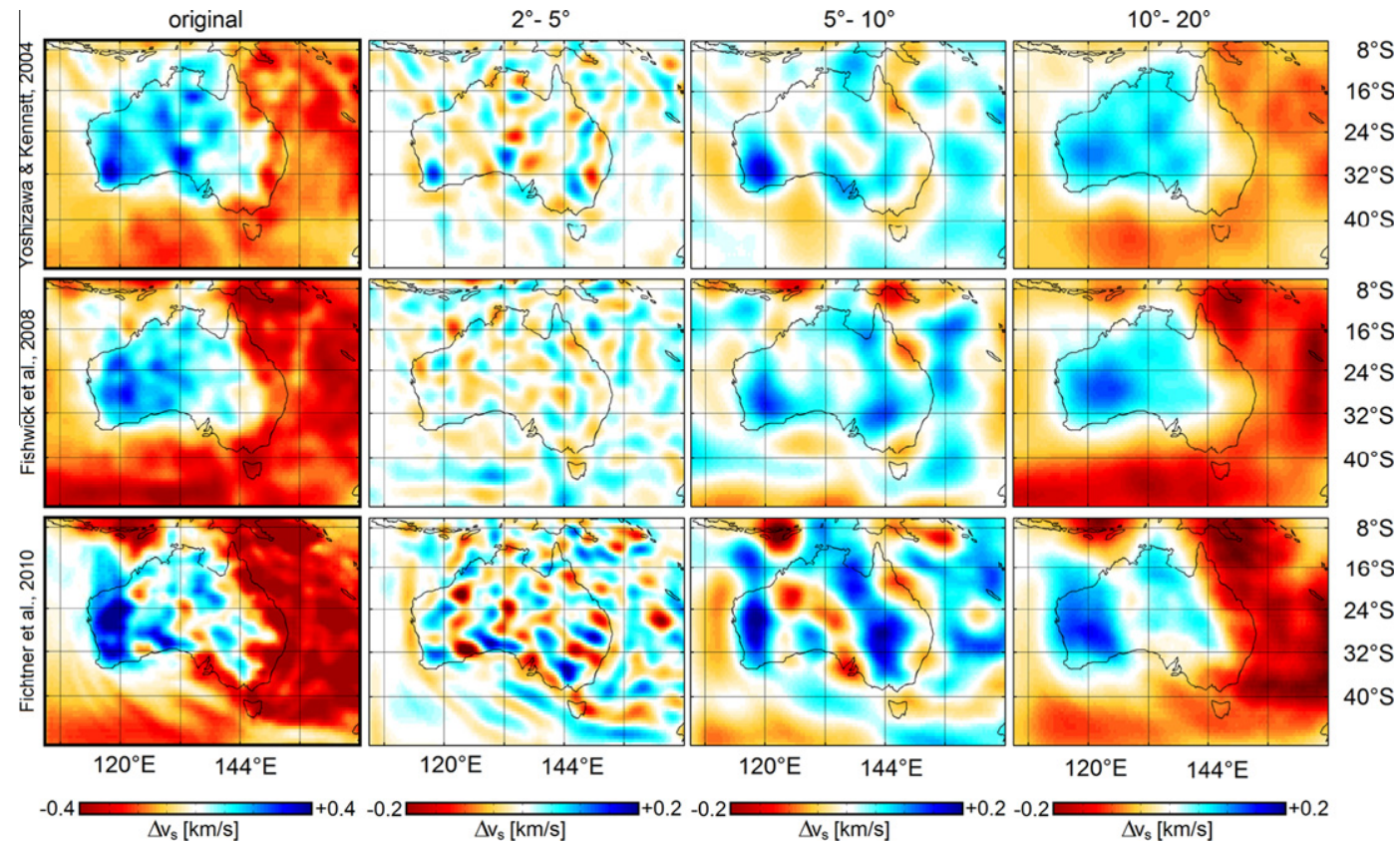

$16^{\circ} \mathrm{S}$

$24^{\circ} \mathrm{S}$

$32^{\circ} \mathrm{S}$

$40^{\circ} \mathrm{S}$

$8^{\circ} \mathrm{S}$
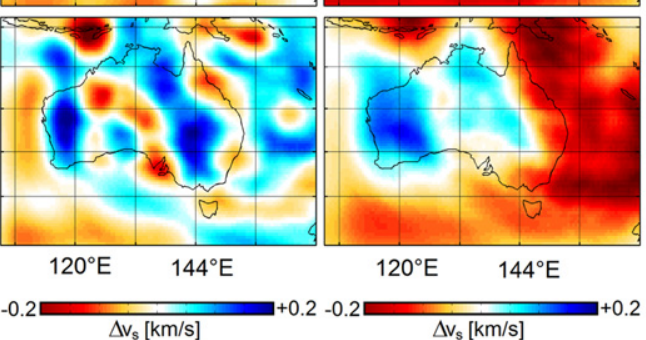

$16^{\circ} \mathrm{S}$

$24^{\circ} \mathrm{S}$

$32^{\circ} \mathrm{S}$

$40^{\circ} \mathrm{S}$

Fig. 4. Comparison of the SV velocity models by Yoshizawa and Kennett (2004), Fishwick et al. (2008) and Fichtner et al. (2010) at $100 \mathrm{~km}$ depth and in different wavelength ranges. The absolute perturbations are relative to the spherically symmetric model ak135 (Kennett et al., 1995).

resolution issue of an insufficient azimuthal distribution of the path coverage. Furthermore, some of the dissimilarity at shortest wavelengths may be expected as the average horizontal resolution of the models is estimated to be around $3-5^{\circ}$. However, since the heterogeneities are sufficiently mild to ensure that all three methods operated within their respective range of validity, we hypothesise that differences in the data, regularisation and data errors are the most important factors.

\section{Discussion and conclusions}

We present a method for the design and efficient application of spatial bandpass filters on sections of the sphere that can be used for the analysis of high-resolution multi-scale tomographic images. The method is based on the spherical spline representation of a tomographic model at a specific depth, where Abel-Poisson kernels serve as spline basis. Linear combinations of Abel-Poisson scaling functions can be used to approximate bandpass filters with predefined properties that can then be applied to the splines without explicity solving integrals over the unit sphere.

As illustrated in Section 3, the application of various bandpass filters can be used to determine the strength and distribution of small-scale heterogeneities, to quantify the sharpness of slow-tofast transitions and to identify structural features that are consistently found in independently derived models.

The major advantages of our approach are its computational efficiency and flexibility in the construction of filters with properties that suit specific purposes. Furthermore, the spherical spline representation ensures that the method is applicable to tomographic models defined on irregular grids that often result from resolution-adaptive parametrisations. For instance, a regionalscale model of $10^{\circ} \times 10^{\circ}$ lateral extent and $0.25^{\circ}$ resolution requires $O\left(10^{3}\right)$ spherical splines for its representation. Thus, $O\left(10^{3}\right)$ operations are needed to filter the model using our approach. Spherical harmonics, in contrast, would require $O\left(10^{6}\right)$ coefficients and as many operations for filtering. This advantage of our method becomes more pronounced when the grid spacing is irregular. Including regional or local seismic data may allow us to reduce the grid spacing in certain regions substantially. Such a local refinement leads to a rapid growth in the number of sphericalharmonic coefficients and the corresponding filter operations. The costs of our spherical spline method, however, grow slowly when the grid is refined locally.

That the combination of two lowpass filters effectively acts as a bandpass filter is a well-known property, used in the construction of multi-resolution analyses sensu stricto (e.g. Freeden et al., 1998). However, as illustrated in Fig. 5, the filter characteristics of these two-stage bandpass filters decay slowly outside the desired passband, which frequently leads to an inadequate suppression of the wavelengths that one attempts to eliminate. The superposition of more than two lowpass filters allows us to sharpen the decay of the filter characterisitics outside the passband, though at the expense of losing the property of defining a multiresolution analysis. From a series of numerical experiments such

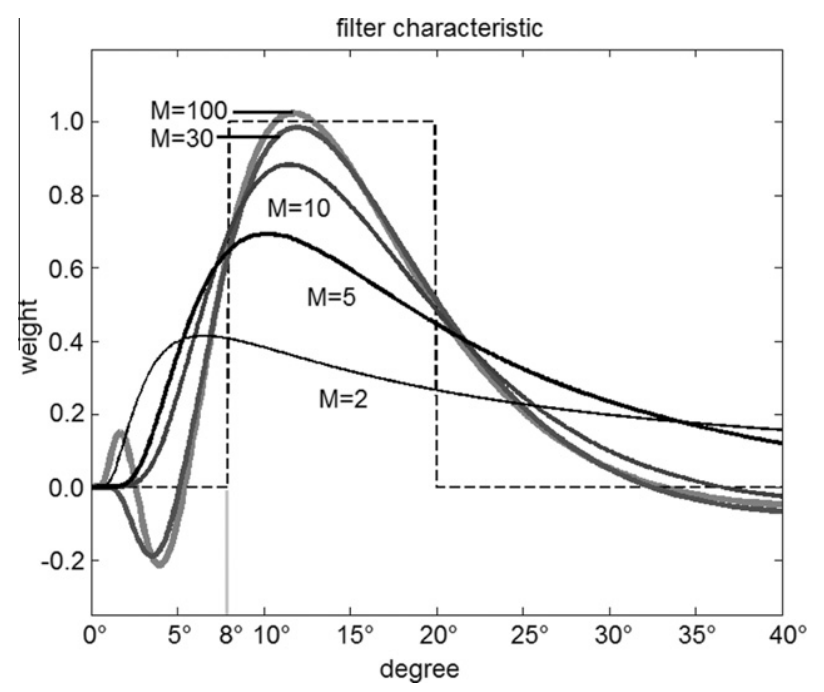

Fig. 5. Filter characteristics of bandpass filters as a function of the number of involved lowpass filters, $M$. The target filter characteristics, shown as dashed curve, are 1 inside and 0 outside the interval $\left[8^{\circ}, 20^{\circ}\right]$. The quality of the approximation increases notably when the number of lowpasses is increased from $M=2$ to $M=5$. However, increasing $M$ from 30 to 100 only leads to minor improvements. 
as the one summarised in Fig. 5, we concluded that the optimal number of combined lowpass filters typically ranges between $M=5$ and $M=10$. For $M<5$, the desired filter characteristics are usually difficult to approximate, and for $M>10$ the computational costs increase, but often without significant improvements in the approximation.

In the interest of simplicity we did not explicity account for the potential restriction to smaller sections of the sphere. Outside the domain where the tomographic model is defined, the spherical spline representation assumes zero values, which can lead to undesirable edge effects when filtering to wavelengths that are close to the width of the spline basis functions. In practice, however, these edge effects are hardly relevant because the resolved, and thus interpretable, length scales are generally much larger than the width of the Abel-Poisson kernels used to represent the model. On the other side of the wavelength spectrum, meaningless results arise from the application of lowpass filters with a cutoff wavelength near to or above the size of the spherical section. Problems with both the shortest and longest wavelengths can be overcome by using spatio-spectrally localised basis functions (e.g. Simons, 2010)

\section{Acknowledgements}

This work arose out of discussions associated with the development of the Australian Seismological Reference Model (AuSREM). Andreas Fichtner would like to thank André Horbach for inspiration and the excellent introduction to spherical splines and filtering. Furthermore, we thank Yann Capdeville and Frederik Simons for discussions that helped to improve the manuscript. We are particularly grateful to Theo van Zessen for maintaining The STIG and GRIT, his little brother. Numerous computations were done on the Huygens IBM p6 supercomputer at SARA Amsterdam. Use of Huygens was sponsored by the National Computing Facilities Foundation (N.C.F.) under the project SH-161-09 with financial support from the Netherlands Organisation for Scientific Research (N.W.O.). Andreas Fichtner was funded by The Netherlands Research Center for Integrated Solid Earth Sciences under project number ISES-MD.5.

\section{Appendix A. Convolution of the Abel-Poisson scaling function with a spherical spline}

In this appendix we proof Eq. (7). As a preparatory step, we note the Legendre expansion of the Abel-Poisson kernel introduced in Eq. (2):

$K_{h}(\mathbf{x}, \mathbf{y})=\sum_{n=0}^{\infty} \frac{2 n+1}{4 \pi} h^{n} P_{n}(\mathbf{x} \cdot \mathbf{y})$.

Eq. (A.1) implies that the Legendre coefficients $K_{h, n}$ of the AbelPoisson kernel are equal to $h^{n}$. Furthermore, we recall Funk-Hecke's relation that holds for any scalar function $G$ defined on the interval $[-1,1]$,

$\int_{\Omega} G(\mathbf{x} \cdot \mathbf{y}) P_{n}(\mathbf{z} \cdot \mathbf{y}) d^{3} \mathbf{y}=G_{n} P_{n}(\mathbf{x} \cdot \mathbf{z})$,

where $P_{n}$ is the Legendre polynomial of degree $n$, and $G_{n}$ is the degree $n$ Legendre coefficient of G. To derive Eq. (7) we write the spherical convolution of the Abel-Poisson scaling function with a spherical spline explicitly in terms of the spline coefficients $v_{i}$ :

$(\varphi * v)(\mathbf{x})=\sum_{i=1}^{N} v_{i}\left(\phi * K_{h}\left(\mathbf{x}_{i},.\right)\right)(\mathbf{x})$.

Expanding both $\phi$ and $K_{h}$ into Legendre series, transforms Eq. (A.3) into

$$
\begin{aligned}
(\varphi * v)(\mathbf{x})= & \sum_{i=1}^{N} v_{i}\left[\left(\sum_{n=0}^{\infty} \frac{2 n+1}{4 \pi} \phi_{n} P_{n}\right) *\left(\sum_{m=0}^{\infty} \frac{2 m+1}{4 \pi} K_{h, m} P_{m}\right)\right](\mathbf{x}) \\
= & \sum_{i=1}^{N} \sum_{n=0}^{\infty} \sum_{m=0}^{\infty} \frac{2 n+1}{4 \pi} \frac{2 m+1}{4 \pi} v_{i} \phi_{n} K_{h, m} \\
& \times \int_{\Omega} P_{n}(\mathbf{x} \cdot \mathbf{y}) P_{m}\left(\mathbf{x}_{i} \cdot \mathbf{y}\right) d^{3} \mathbf{y}
\end{aligned}
$$

Upon invoking Funk-Hecke's relation (Eq. A.2), Eq. (A.4) simplifies to

$$
\begin{aligned}
(\varphi * v)(\mathbf{x})= & \sum_{i=1}^{N} \sum_{n=0}^{\infty} \sum_{m=0}^{\infty} \frac{2 n+1}{4 \pi} \frac{2 m+1}{4 \pi} \\
& \times v_{i} \phi_{n} K_{h, m} \frac{4 \pi}{2 m+1} \delta_{n m} P_{m}\left(\mathbf{x}_{i}, \mathbf{x}\right) \\
= & \sum_{i=1}^{N} \sum_{n=0}^{\infty} \frac{2 n+1}{4 \pi} v_{i} \phi_{n} K_{h, n} P_{n}\left(\mathbf{x}_{i} \cdot \mathbf{x}\right) .
\end{aligned}
$$

Introducing the Legendre coefficients $\phi_{n}=g^{n}$ and $K_{h, n}=h^{n}$, yields

$(\varphi * v)(\mathbf{x})=\sum_{i=1}^{N} \sum_{n=0}^{\infty} \frac{2 n+1}{4 \pi} v_{i}(g h)^{n} P_{n}\left(\mathbf{x}_{i} \cdot \mathbf{x}\right)$.

According to Eq. (A.1), the sum over $n$ can be evaluated explicitly. It yields the Abel-Poisson kernel with the parameter $g h$ :

$$
\begin{aligned}
(\varphi * v)(\mathbf{x}) & =\sum_{i=1}^{N} v_{i} K_{g h}\left(\mathbf{x}_{i}, \mathbf{x}\right), \quad K_{g h}(\mathbf{x}, \mathbf{y}) \\
& =\frac{1}{4 \pi} \frac{1-(g h)^{2}}{\left[1+(g h)^{2}-2 g h \mathbf{x} \cdot \mathbf{y}\right]^{3 / 2}} .
\end{aligned}
$$

This concludes the proof.

\section{References}

Becker, T.W., Boschi, L., 2002. A comparison of tomographic and geodynamic mantle models. Geochem. Geophys. Geosyst. 3, 10.129/2001GC000168.

Cara, M., Lévèque, J.J., 1987. Waveform inversion using secondary observables. Geophys. Res. Lett. 14, 1046-1049.

Della Mora, S., Boschi, L., Tackley, P.J., Nakagawa, T., Giardini, D., 2011. Low seismic resolution cannot explain S/P decorrelation in the lower mantle. Geophys. Res. Lett. 38. doi:10.1029/2011GL047559.

Fichtner, A., Kennett, B.L.N., Igel, H., Bunge, H.-P., 2009. Full seismic waveform tomography for upper-mantle structure in the Australasian region using adjoint methods. Geophys. J. Int. 179, 1703-1725.

Fichtner, A. Kennett, B.L.N., Igel, H., Bunge, H.-P. 2010. Full waveform tomography for radially anisotropic structure: new insights into present and pasts states of the Australasian upper mantle. Earth Planet. Sci. Lett. 290, 270-280.

Fichtner, A., Trampert, J., 2011. Hessian kernels of seismic data functionals based upon adjoint techniques. Geophys. J. Int. 185, 775-798.

Fishwick, S., 2006. Gradient maps: a tool in the interpretation of tomographic images. Phys. Earth Planet. Int. 156, 152-157.

Fishwick, S, Heintz, M., Kennett, B.L.N., Reading A.M., Yoshizawa, K., 2008. Steps in lithospheric thickness within eastern Australia, evidence from surface wave tomography. Tectonics 27, 10.129/2007TC002116.

Freeden, W., Gervens, T., Schreiner, M., 1998. Constructive Approximation on the Sphere. Oxford Science Publications. Clarendon Press.

Hernlund, J.W., Houser, C., 2008. On the statistical distribution of seismic velocities in Earth's deep mantle. Earth Planet. Sci. Lett. 265, 423-437.

Kennett, B., Salmon, M., Saygin, E., Group, A.W., 2011. AusMoho: the variation of crustal thickness in Australia. Geophys. J. Int. 0000, 0000-0000.

Kennett, B.L.N., Engdahl, E.R., Buland, R., 1995. Constraints on seismic velocities in the Earth from traveltimes. Geophys. J. Int. 122, 108-124.

Kirkpatrick, S., Gelatt, C.D., Vecchi, M.P., 1983. Optimisation by simulated annealing. Science 220, 671-680.

Lebedev, S., van der Hilst, R.D., 2008. Global upper-mantle tomography with the automated multimode inversion of surface and S-wave forms. Geophys. J. Int. 173, 505-518.

Lei, J., Zhao, D., Su, Y., 2009. Insight into the origin of the Tengchong intraplate volcano and seismotectonics in southwest China from local and teleseismic data. J. Geophys. Res. 115. doi:10.1029/2008JB005881.

Molinari, I., Morelli, A., 2011. EPcrust: a reference crustal model for the European plate. Geophys. J. Int. 185, 352-364. 
Ritsema, J., Deuss, A., van Heijst, H.J., Woodhouse, J.H., 2011. S40RTS: a degree-40 shear-velocity model for the mantle from new Rayleigh wave dispersion, teleseismic traveltime and normal-mode splitting function measurements. Geophys. J. Int. 184, 1223-1236.

Schivardi, R., Morelli, A., 2011. Epmantle: a 3-D transversely isotropic model of the upper mantle under the european plate. Geophys. J. Int. 185, 469-484.

Simons, F.J., 2010. Slepian functions and their use in signal estimation and spectral analysis. J. Int. 187, 969-988.
Simons, F.J., Loris, I., Nolet, G., Daubechis, I.C., Voronin, S., Judd, J.S., Vetter, P.A., Charlty, J., Vonesch, C., 2010. Solving or resolving global tomographic models with spherical wavelets, and the scale and sparsity of seismic heterogeneity. Geophys. J. Int.

Yoshizawa, K., Kennett, B.L.N., 2004. Multimode surface wave tomography for the australian region using a three-stage approach incorporating finite frequency effects. J. Geophys. Res. 109. doi:10.1029/2002JB002254. 